\title{
Minimally invasive endoscopic treatment of gastric leiomyosarcoma: A rare case report and review of literature
}

guixiang kong ( $\sim 905559613 @ q q . c o m$ )

Lanzhou University Second Hospital https://orcid.org/0000-0001-5193-0716

xiaolu Su

Lanzhou University Second Hospital

xiaojun Huang

Lanzhou University Second Hospital

\section{Case report}

Keywords: Gastric leiomyosarcoma, Endoscopy, Minimally invasive treatment

Posted Date: October 28th, 2021

DOI: https://doi.org/10.21203/rs.3.rs-995117/v1

License: (9) (1) This work is licensed under a Creative Commons Attribution 4.0 International License. Read Full License 


\section{Abstract}

Gastrointestinal leiomyosarcoma remains relatively rare,accounting for only $1 \%$ of gastric tumors, since the use of $c$-kit staining was first described in 1998 to differentiate gastrointestinal stromal tumors. In this case report,we described the clinical manifestations,endoscopic features, and pathological immunohistochemical features of the gastric leiomyosarcoma. We found that endoscopic ultrasonography has great value in the diagnosis of gastric leiomyosarcoma,and that minimally invasive endoscopic treatment is safe and effective.Particularly,this case highlights that the early-stage lesions can be treated with minimally invasive endoscopic treatment with good tolerance and satisfactory clinical results. Endoscopic submucosal dissection is thus effective for treating gastric leiomyosarcoma,with promising prospects for clinical application.

\section{Introduction}

Gastric leiomyosarcoma (LMS) is a rare gastrointestinal tumor,with limited studies reporting on its clinical imaging and endoscopy findings. Thus, subepithelial tumors,such as LMS and gastrointestinal stromal tumors (GISTs), are difficult to differentiate.Especially for endoscopic manifestations, them are subepithelial tumors with solid round appearance,normal surface covering mucosa,tough or hard texture,and originated from muscularis mucosae to muscularis propria.Large tumors may present with ulcers and other manifestations.In general,only deep biopsy or endoscopic ultrasound-guided fine-needle aspiration(EUS-FNA) tissue can be differentiated by immunohistochemical detection.As a result,pathological immunohistochemistry has become the key to differential diagnosis,and endoscopic ultrasonography(EUS) can be of great value in its diagnosis.In particular,minimally invasive endoscopic therapy has a high safety profile and satisfactory effect;based on the case described herein,it is promising for clinical application. Herein,we provide the endoscopic image findings and describe current practices in endoscopic diagnosis and minimally invasive treatment both at home and abroad.

\section{Case Report}

A 63-year-old female patient visited our hospital with chief complaints of "epigastric pain and discomfort for 2 months."The patient underwent esophagogastroduodenoscopy (EGD) for screening and a mass was detected at the gastric body.Upon examination,we noted that she had a poor diet and had lost about $2 \mathrm{~kg}$ of body weight. The patient had a 15-year history of hypertension that was well-controlled with oral drugs.

At hospitalization,the patient vital signs were stable.There was mild tenderness in her left upper abdomen.Laboratory tests showed no abnormalities.Abdominal contrast-enhanced CT found slight thickening of the gastric body wall.Repeat EGD revealed a polyp-like mass protruding into the gastric lumen was seen,indicating roughness and unevenness with ulcer formation, with mucosal aggregation,and when touched with biopsy forceps, the mass was found to be hard and fixed(Fig. 1A).EUS revealed a round protuberant mass that originated from the muscularis mucosa on the greater curvature of the gastric body,with a maximum diameter of $16 \mathrm{~mm}$

(Fig. 2).Considering the tumor was mesenchymal tissue origin,and after pursuing the medical history,it was found that the lesion changed significantly within a short period of time,with potential malignancy.In addition,the lesion could be completely resected.At the same time,the low positive rate of ordinary endoscopic biopsy was also considered,and the cost of EUS to take biopsy was expensive but not fully confirmed,therefore, after full communication with the patient and exclusion of contraindications, the patient successfully underwent diagnostic endoscopic submucosal dissection (ESD) surgery(Fig. 1B-D). 
The postoperative pathological examination of the surgical specimens (Fig. 3) show

pleomorphic,spindled,eosinophilic,and mitotic figures in the tumor cells,with approximately 10 mitoses per highpower fields,and abundant blood vessels around the tumor.The immunohistochemical staining panel (Fig. 3) included vimentin (+), desmin (+), epithelial membrane antigen (-),CD117 (-),DOG-1 (-),S100 (-), smooth muscle actin $(-)$, synapsin (-),CD56 (-), p63 (-),P40 (-),thyroid transcription factor-1(-),CK5/6 (-),C-reactive protein (-),transducer-like enhancer of split 1(-),HMB45(-),SOX10(-),PMS-2(+),MSH-2 (+),MSH-6(+), and Ki67 (40\%).Vascular endothelial cells were immunostained with anti-CD34 antibody.Based on these results,LMS was considered as the diagnosis;however,GIST was not excluded.Subsequently,molecular detection showed that exons 9, 11, 13,and 17 of the $c$-kit gene and exons 12 and 18 of the PDGFRA gene were of the wild type. Since this was a rare case,the specimens were taken to two other authoritative pathological institutions for consultation,and a diagnosis of LMS was finally established. The patient recovered well and was discharged from the hospital.

After 20 months of follow-up,the patient remains in good condition,and follow-up gastroscopy and enhanced CT of the abdomen indicated no relapse.

\section{Discussion}

Since $c$-kit staining was first described in 1998,which led to the recognition of GISTs;ever since,gastrointestinal LMS has been a relatively rarely diagnosed disease. It has been reported that gastrointestinal LMS accounts for less than $0.5 \%$ of all mesenchymal tumors. The most commonly site in LMS is the colon,while the least site is the stomach[1]. Yamamoto et al.[2] reported 55 cases of gastrointestinal LMS,and only four cases occurred in the stomach, indicating that the stomach is the least common site.In March 2018,Japan published the first report in English[3],describing the case of a patient with gastric LMS treated by ESD,thereby indicating the safety and efficacy of the procedure.In March 2020,Rajat et al.[4],after a comprehensive search of multiple databases and additional sources,reported the cases of 16 patients with gastric LMS.The mean age of LMS onset in these patients was 52 years,and the ratio of males to females was equal.The most common symptom in these patients was gastrointestinal bleeding, followed by abdominal pain,weight loss,and gastroesophageal reflux, with the average tumor size being $4.80 \mathrm{~cm}$. The tumors occur most frequently in the body of the stomach, especially in the upper part of the gastric body,followed by the antrum and fundus,and appear as either a solitary lesion or multiple lesions. These lesions rarely affect gastrointestinal function in their early stage and are often treated in the middle and late stages,wherein the patient typically presents with hemorrhage,anemia,and/or an epigastric mass. In June 2021, Toru Takagi et al.[5] summarized that only two cases were originated from the muscularis mucosae(Table1).However, due to the lack of known clinical manifestations, the tumors can be easily misdiagnosed as a peptic ulcer, gastric cancer,subepithelial tumors-such as stromal tumors-and other diseases. The lack of understanding of the disease is the main cause of overlooked diagnosis and misdiagnosis,while timely diagnosis is the main factor affecting the survival rate of patients with gastric LMS.

Table 1

Cases of leiomyosarcoma of the stomach derived from muscularis mucosa reported since 2000s

\begin{tabular}{|lllllllll|}
\hline $\begin{array}{l}\text { Case } \\
\text { no }\end{array}$ & Age/sex & Location & $\begin{array}{l}\text { Size } \\
\text { (cm) }\end{array}$ & $\begin{array}{l}\text { Endoscopic } \\
\text { appearance }\end{array}$ & $\begin{array}{l}\text { Histological } \\
\text { location }\end{array}$ & Treatment & Outcome & References \\
\hline 1 & $72 / \mathrm{F}$ & $\begin{array}{l}\text { gastric } \\
\text { body }\end{array}$ & 2.5 & $\begin{array}{l}\text { Polypoid } \\
\text { lesion }\end{array}$ & $\begin{array}{l}\text { Muscularis } \\
\text { mucosae }\end{array}$ & $\begin{array}{l}\text { Endoscopic } \\
\text { resection }\end{array}$ & $\begin{array}{l}\text { not } \\
\text { mentioned }\end{array}$ & $\begin{array}{l}\text { Agaimy } \\
\text { A,et al }\end{array}$ \\
\hline 2 & $26 / M$ & $\begin{array}{l}\text { gastric } \\
\text { fundus }\end{array}$ & 7.2 & $\begin{array}{l}\text { Large } \\
\text { lobulated }\end{array}$ & $\begin{array}{l}\text { Muscularis } \\
\text { mucosae }\end{array}$ & $\begin{array}{l}\text { Surgical } \\
\text { resection }\end{array}$ & $\begin{array}{l}\text { Alive (1 } \\
\text { month) }\end{array}$ & $\begin{array}{l}\text { Aggarwal } \\
\text { G,et al }\end{array}$ \\
\hline
\end{tabular}


EGD is the main method used for the diagnosis of the disease,and the shape of the mass can be directly observed under dynamic conditions.Polyp-like masses protruding into the gastric lumen can often be observed by EGD. Often,the surface of the mass is rough,with ulcers,erosion, and bleeding,and the formation of gouge- or navel-like ulcers is significant for diagnosis.A biopsy can be performed preoperatively for an immunohistochemical diagnosis,which provides a reliable basis for treatment[6].In the present case,when touched with biopsy forceps,the mass was tough or hard and relatively fixed.After full discussion this lesion was removed directly by ESD.Pathological immunohistochemistry of the current patient indicated that the mass was CD117 (-) and DOG-1 $(-)$,and molecular detection indicated the presence of wildtype $c$-kit and PDGFRA genes, which ruled out the possibility of GIST.After consideration of other characteristics,such as vimentin(+),desmin(+), and cell morphology, the case was finally diagnosed as LMS.Of course,LMS does not require molecular testing,which is designed to rule out wild-type GISTs. The important immunostaining of LMS are CD117,DOG-1,CD34,desmin,vimentin,smooth muscle actin,synapsin and Ki67,etc.

In recent years,EUS has become highly valued for diagnostics.It can clearly show the structural characteristics of each layer of the gastric wall to help understand the depth and extent of tumor invasion;in particular, biopsies of the submucosal mass can be performed under EUS guidance, with a diagnostic confirmation rate of more than $90 \%$.It also has good sensitivity and specificity for the diagnosis of gastric LMS[7].EUS can also help visualize the blood vessels around the lesion,which can provide justification for and necessary guidance during endoscopic resection[8].At the same time,it can provide more targeted and informed postoperative follow-up[9].

Gastric LMS is not sensitive to radiotherapy or chemotherapy,but its prognosis is better than that of gastric cancer[10].Because clinical cases are rare,a large sample of data and standardized treatment do not exist to date. Partial or total gastrectomy is the primary treatment for gastric LMS. According to the current literature and the characteristics of the EUS images from the present patient,gastric LMS can originate from superficial structures such as the muscularis mucosa layer.Additionally,lymph node metastasis appears to be rare,so ESD has several advantages over traditional surgery, such as less trauma,faster recovery, and lower costs.Importantly,patients' postoperative quality of life is not lowered.Moreover, compared with ordinary biopsy or EUS-FNA,complete resection of the lesion can greatly improve the accuracy of differential pathological diagnosis due to larger tissue specimens for immunohistochemistry evaluation and molecular detection.In the present case,ESD was used to remove the tumor,achieving radical resection with a good patient prognosis as the follow-up continues to date. Of course,the flaw of our concern is that this case has been followed up for less than three years.But This case and a few other reports strongly demonstrate the feasibility of ESD of LMS.

In short,although gastric LMS is rare,with a comprehensive evaluation and clear diagnosis,early-stage lesions can be treated with minimally invasive endoscopic surgery with high safety,good tolerance,and satisfactory clinical results. This shows that minimally invasive endoscopic resection is an effective method for the treatment of LMS and has promising prospects for clinical application.At the same time,this report also provides valuable EGD and EUS data for primary gastric LMS,adding details about the clinical diagnosis to the current literature.

\section{Declarations}

\section{Informed consent statement}

Informed written consent was obtained from the patient for publication of this report and any accompanying images. 


\section{Conflict-of-interest statement}

The authors declare that they have no conflict of interest.

\section{Author contributions}

Gui-Xiang Kong was the patient's gastroenterologist, reviewed the literature and contributed to manuscript drafting;Xiao-Lu Su analyzed and interpreted the pathological findings; Xiao-Jun Huang was responsible for the revision of the manuscript for important intellectual content; all authors issued final approval for the version to be submitted.

\section{References}

1. Miettinen M,Fetsch JF.Evaluation of biological potential of smooth muscle tumours. Histopathology.2006;48(1):97-105.

2. Yamamoto $\mathrm{H}, \mathrm{Handa} \mathrm{M}$, Tobo T,et al. Clinicopathological features of primary leiomyosarcoma of the gastrointestinal tract following recognition of gastrointestinal stromal tumours. Histopathology. 2013;63(2):194-207.

3. Sato T, Akahoshi K, Tomoeda N,et al. Leiomyosarcoma of the stomach treated by endoscopic submucosal dissection. Clin J Gastroenterol. 2018;11(4):291-6.

4. Garg R. AlRajjal A,Berri R,Barawi M.Primary Gastric Leiomyosarcoma: a Case Report and Review of the Literature. J Gastrointest Cancer. 2020;51(1):335-40.

5. Takagi T, Saito S, Yokota S,et al. Laparoscopic and endoscopic cooperative surgery for leiomyosarcoma of the stomach: a case report with a review of the literature. Surg Case Rep. 2021;7(1):146-53.

6. Wang Y,Zhou X.Newly recognized gastrointestinal mesenchymal tumor.Journal of Clinical and Experimental Pathology.2015; (8): 841-845.

7. Wu A, Li R, Shi D,et al. Diagnostic value of endoscopic ultrasonography for submucosal tumors of upper gastrointestinal tract. Chinese Journal of Gastrointestinal surgery. 2015;18(11):1136-8.

8. Soufi M. Errougani A,Chekkof RM.Primary gastric leiomyosarcoma in young revealed by a massive hematemesis. J Gastrointest Cancer. 2009;40(1-2):69-72.

9. Chang L, Zhang K, Zhang M,et al. Diagnosis, treatment and follow-up of submucosal tumors of upper digestive tract. World Journal of Chinese digestion. 2016;24(5):765-74.

10. Shi Y,Jia B.Progress in the treatment of soft tissue sarcoma. Chinese Oncology Clinic.2014; (24): 1556-1560.

\section{Figures}




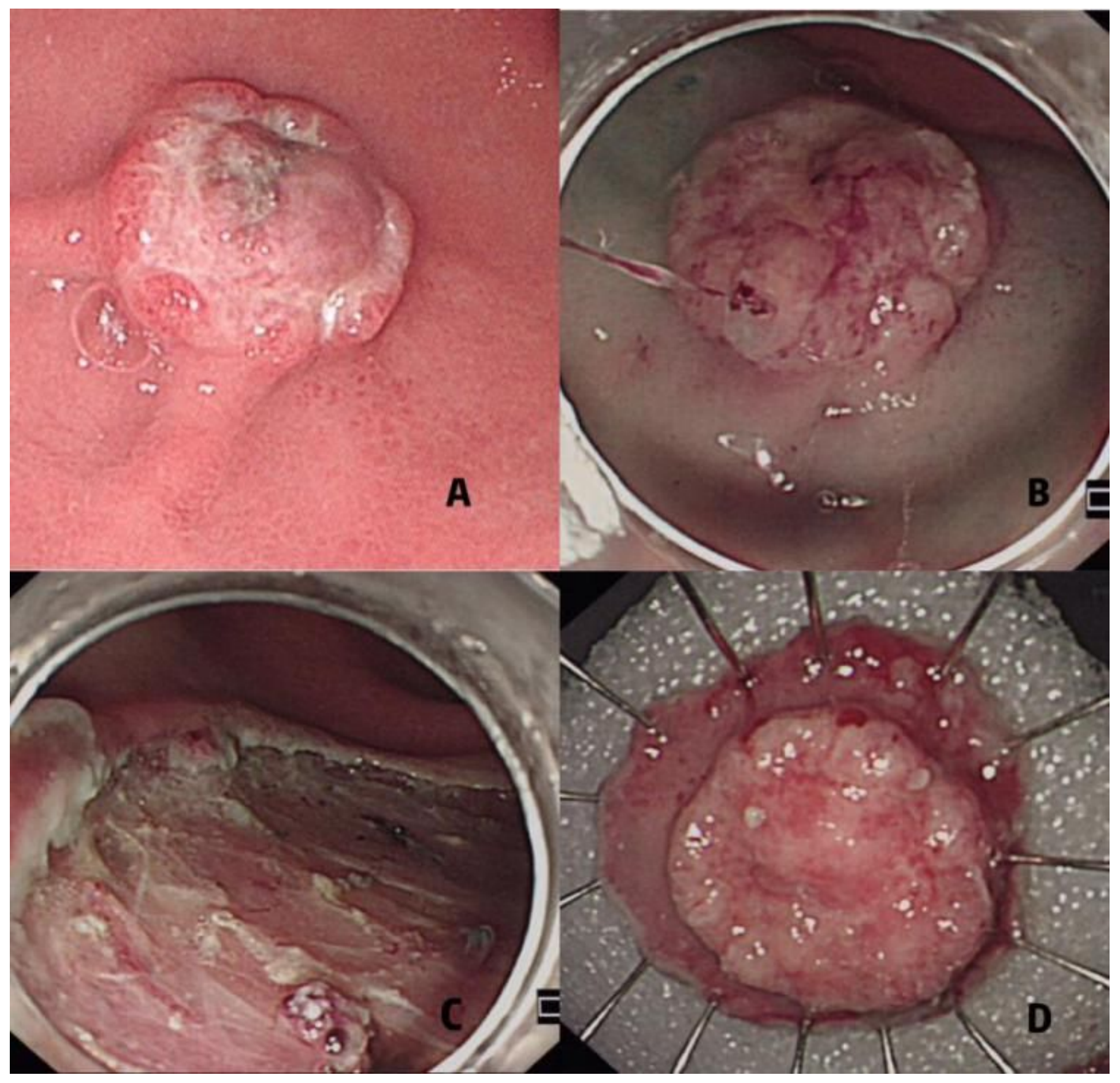

Figure 1

Endoscopic features :A protruding tumor is in the gastric lumen with the ulceration on the surface and it has a positive elevation sign after intraoperativeafter submucosal injection. Eventually,the tumor is completely removed by ESD surgery. 


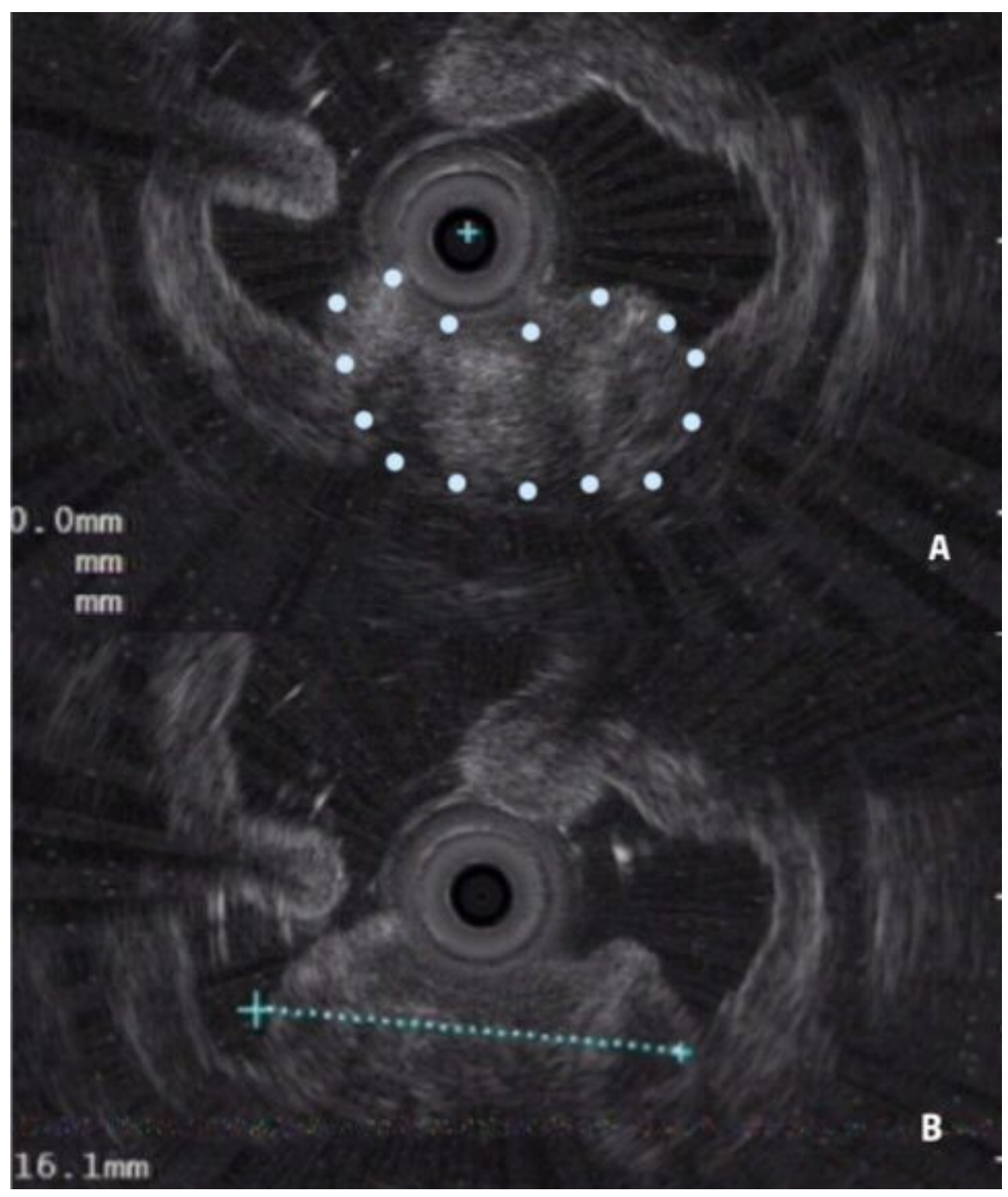

\section{Figure 2}

Features under endoscopic ultrasonography :The lesion originates from the second muscularis mucosa layer, and has an uneven internal echo and cystic space.The white dots are the outline of the tumor. 


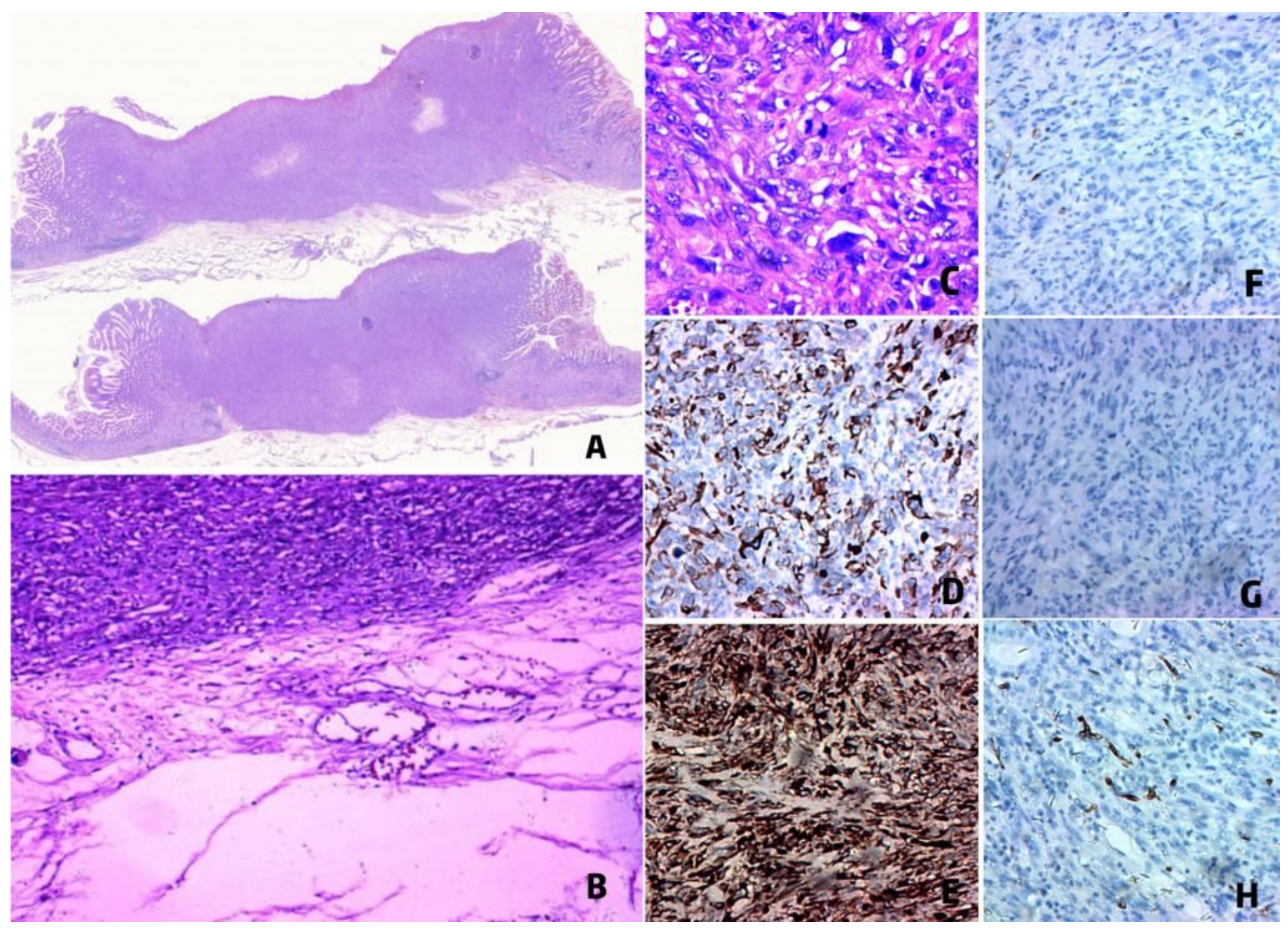

Figure 3

Postoperative pathological findings A: hematoxylin and eosin (HE) stain; B: 40x HE staining; C: 400x HE staining; D: tumor cells are desmin positive; E: tumor cells are vimentin positive; F: tumor cells are CD117 negative; G: tumor cells are DOG-1 negative; H:tumor cells are CD34 negative

\section{Supplementary Files}

This is a list of supplementary files associated with this preprint. Click to download.

- CertificateofeditingKONGG2264onlplcl.pdf 